\title{
Influence of Combustible Dimension and Density on Heat Release Rate Part 2 Relation between Combustible Dimension, Maximum Heat Release Rate and Duration of Maximum Burning
}

\author{
Norichika Kakae ${ }^{1}$, Tsuneto Tsuchihashi ${ }^{2}$, Yoshiaki Tanaka ${ }^{2}$, Yoshifumi Ohmiya ${ }^{1}$ \\ and Kazunori Harada ${ }^{3}$ \\ ${ }^{1}$ Tokyo University of Science \\ ${ }^{2}$ General Building Research Corporation of Japan \\ ${ }^{3}$ Kyoto University
}

Keywords : Burning Behavior, Heat Release Rate, Maximum Heat Release Rate, Duration of Burning, Polyurethane

\section{INTRODUCTION}

It is important to understand the burning behavior of the familiar flammable materials used in the various aspects of the fire safety design of a building, such as evacuation safety. The time history of the heat release rate can be regarded as an indicator of the spreading speed of the fire. In the past, the heat release rate of various flammables has been measured,[1] but it has not yet been confirmed how time history of the heat release rate changes with respect to the material and the dimension standard.

Therefore, a burning experiment was conducted by using polyurethane mattresses with different dimensions and densities, and this report demonstrates the findings regarding the relation between the time history on one hand and the maximum heat release rate, the maximum scale burning and the dimension and density of the test object on the other.

\section{EXPERIMENTAL METHOD}

\subsection{Test objects}

The long-side length of the test object used in this experiment was fixed at approximately $900 \mathrm{~mm}$, the thickness was changed from $40 \mathrm{~mm}$ to $420 \mathrm{~mm}$, and the short-side length was also changed from $200 \mathrm{~mm}$ to approximately $650 \mathrm{~mm}$. Two density values were used: $12.8 \mathrm{~kg} / \mathrm{m}^{3}$ (low density) and $18.5 \mathrm{~kg} / \mathrm{m}^{3}$ (high density). Table 1 provides the details of the test object. 
Table 1 List of the test objects used in this experiment

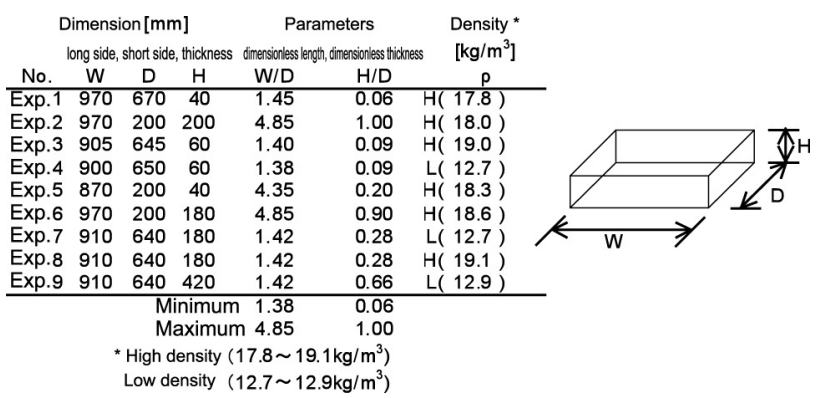

\subsection{Measurement parameters}

This experiment used an oxygen consumption calorimeter in General Building Research Corporation of Japan. The test object was placed below the smoke-collecting hood and a fire was ignited at the center of the upper surface. The flow rate of the combustion gases and the gas concentration $\left(\mathrm{O}_{2}, \mathrm{CO}_{2}, \mathrm{CO}\right)$ were measured at 3 second intervals, and the heat release rate was calculated with the use of the oxygen consumption. Also, in order to obtain the time history of the burning area, two video cameras were installed for the purpose of capturing bidirectional images from the top surface and the lateral side. Other details relating to the measurement parameters can be found in a previous report.[2]

\section{EXPERIMENTAL RESULTS AND DISCUSSION}

As shown in Figure 1, the maximum heat release rate $\left(Q_{\max }\right)$, the time when it reaches $90 \%$ of the maximum heat release rate $\left(t_{A}\right)$, and the time when it becomes lower than $90 \%$ of the maximum heat release rate $\left(t_{B}\right)$ were calculated from the heat release rate curve obtained from this experiment, and the duration of the maximum scale burning was defined as. $\tau_{\max }=t_{B}{ }^{-} t_{A}$.

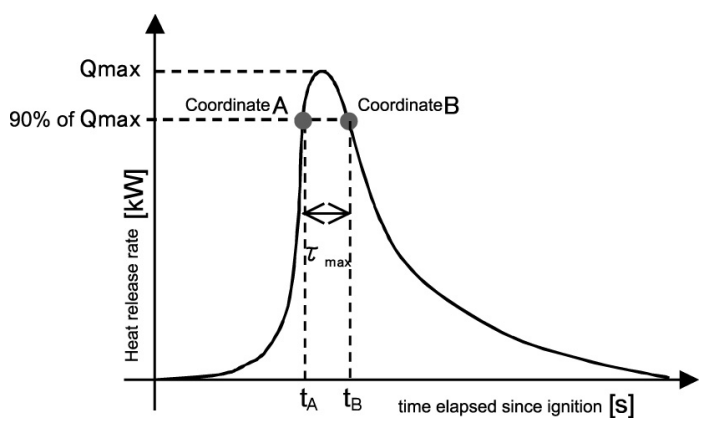

Figure 1 Coordinates on the heat release rate curve

\subsection{Maximum heat release rate}

At the time when the heat release rate reached its maximum, the upper surface of the 
thinner material was mostly engulfed in flames, while in the case of the thicker material both the upper surface and the lateral side were totally engulfed in flames. Given this factor, the size of the burned area at the time when the heat release rate reached its maximum was obtained, and its relation with the initial exposed area is shown in Figure 2. According to Figure 2, the maximum heat release rate increases as the exposed area increases when the initial exposed area is small, but the increase rate becomes lower when the exposed area becomes large. This is attributed to the fact that there is no exposed surface at the time when the heat release rate reached its maximum in the initial condition.

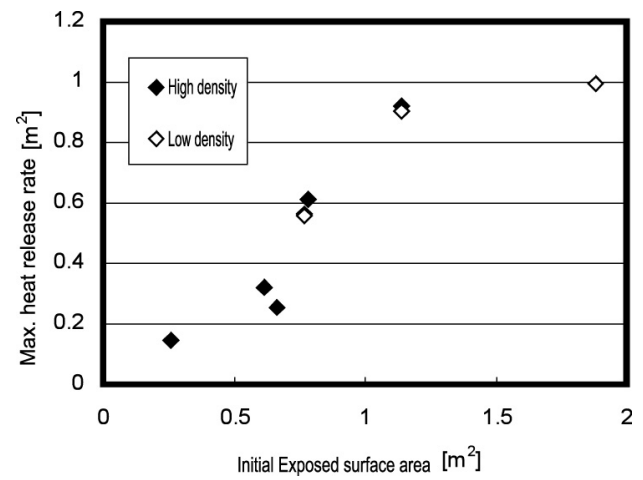

Figure 2 Relation between the maximum heat release rate and the initial exposed surface area

Another finding is that the maximum heat release rate is severely influenced by the kindling phenomenon generated by the instillation of the lateral side. Therefore, separate discussions have been made with respect to whether or not instillation occurred. In the case where no instillation occurred (Experiments 1, 3, 4, 5 and 6), the heat release rate reached its maximum at the time when the upper surface was mostly engulfed in flames. The relationship between the upper surface and the maximum heat release rate is directly proportional and the proportionality coefficient (the heat release rate per unit area) is approximately $280 \mathrm{~kW} / \mathrm{m}^{2}$.

In the case where instillation occurred, it was generated when the burning had progressed downward to a distance $Z_{\text {fall }}$ from the upper surface and the lateral side. The heat release rate reached its maximum at the time when the lateral side was completely engulfed in flames. Given this factor, the following equation was used to obtain the representative area at the time when the heat release rate reaches its maximum.

$$
\text { Representative area }=W D+2(W+D)\left(H+z_{\text {fall }}\right)
$$

Figure 3 is a graph which shows the relation between the representative area and the maximum heat release rate. According to Figure 3, the maximum heat release rate increases as the representative area increases. In Experiments 7 and 8, where the instillation occurred on all four lateral sides, the heat release rate per unit area became $700 \mathrm{~kW} / \mathrm{m}^{2}$. Furthermore, in the cases where the instillation took place for fewer lateral 
sides (two sides in Experiment 9, one side in Experiment 2), the value of the heat release rate with respect to the representative area became smaller when compared with the ones for Experiments 7 and 8. Therefore, under the condition that instillation occurs, the maximum heat release rate is influenced by the number of lateral sides affected by the instillation.
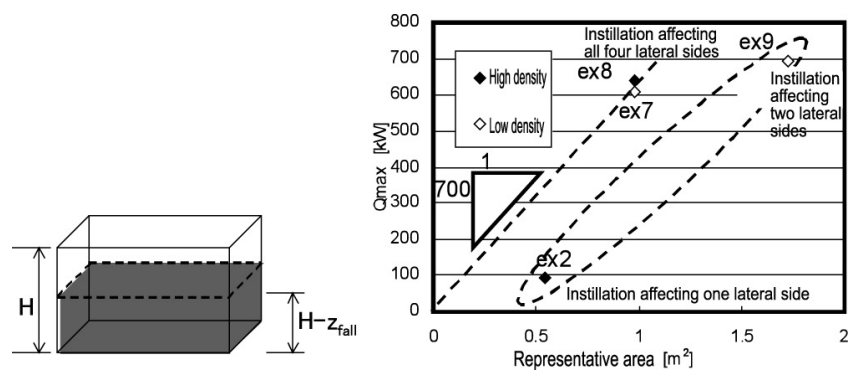

Figure 3 Relation between the maximum heat release rate and the representative area (with instillation)

\subsection{Duration of the maximum scale burning}

Similarly to the maximum heat release rate, the duration of the maximum scale burning is also influenced by the instillation. Therefore, separate discussions are provided with respect to whether or not instillation occurred.

In the case where no instillation occurred, the flame reached the short side of the upper surface at time $t_{A}$, whereby the representative dimension became W/2. On the other hand, the surface was completely burned at time $t_{B}$, and the time at which the burning was complete was different depending on the thickness of the test object. Based on the above points, $H-W / 2$ can be considered to be the main variable of $\tau_{\max }$. In this respect, the relation between $\tau_{\max }$ and $H-W / 2$ is presented in Figure 4. As can be inferred from Figure 4, the burning duration at the maximum scale increases together with the increase of $\mathrm{H}-\mathrm{W} / 2$, however, it is somewhat shorter for the lower density material.

In the case where instillation does occur, assuming that the instillation on the lateral side occurs in the same fashion in each of the Experiments 2, 7, 8 and 9, it can be said that the time $t_{A}$ is determined only by the time needed for the flame to reach the long side of the upper surface. Therefore, $D / 2$ is employed as the representative dimension for the attainment of the time $t_{A}$. On the other hand, the surface is completely burned at $t_{B}$, and the time of completion of the burning process changes depending on the thickness $H$ of the test object. Based on the above considerations, $H-D / 2$ can be regarded as the main variable of $\tau_{\max }$. Therefore, the relation between $\tau_{\max }$ and $H-D / 2$ is given in Figure 5. The figure shows that the burning duration at the maximum scale increases together with the increase of $H-D / 2$.

Comparing the slope with respect to the density, the slope is steeper for the high density material, and the proportion of the slope is the same as the proportion of the density. 
The results from previous measurements using polyurethane mattresses, which are based on the same rules, are given in Figure 5.

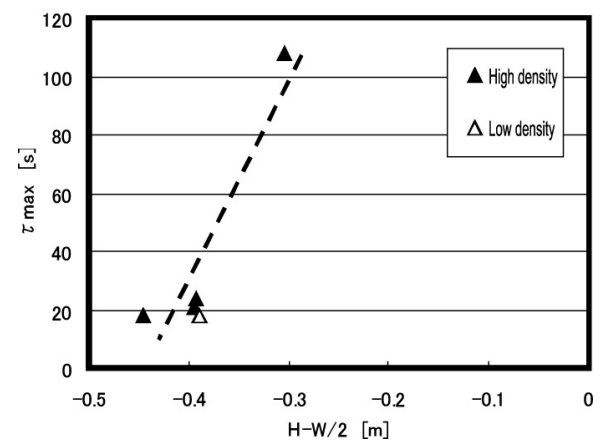

Figure 4 Relation between $\mathrm{H}-\mathrm{W} / 2$ and the duration of the maximum burning (No instillation)

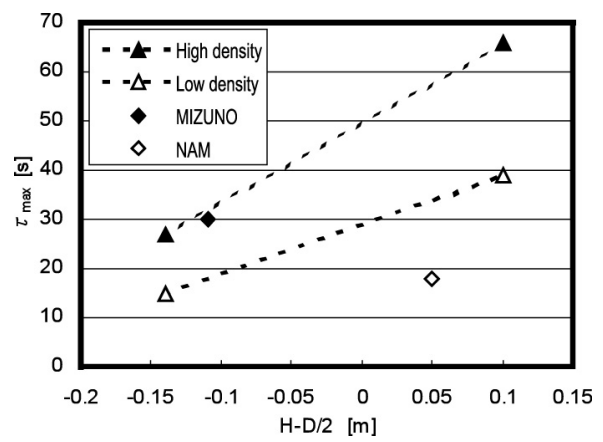

Figure 5 Relation between $H-D / 2$ and the duration of the maximum burning (No instillation)

Similar experiments were conducted by Mizuno et al., with a mattress of $500 \times$ $500 \times 140\left(20.8 \mathrm{~kg} / \mathrm{m}^{3}\right)$, as well as by Nam et al., with a mattress of $1000 \times 500 \times 300$ $\left(118 \mathrm{~kg} / \mathrm{m}^{3}\right)$.[3] As shown in Figure 5, the results of Mizuno's experiment are almost the same in the case of the high density material, which indicates that the considerations about $H-D / 2$ were applicable to that case as well. However, $\tau_{\max }$ is very short in the results of Nam's experiment, since in that case the heat release rate jumped as the time reached the maximum heat release rate. It should be noted that it is difficult to handle the heat release rate if it fluctuates drastically during the experiment.

\section{CONCLUSION}

The maximum heat release rate is proportional to the upper surface area if no instillation occurs. In case instillation does occur, the maximum heat rate is proportional to the size of the surface area with $z_{\text {fall }}$ subtracted from the lateral side. However, the maximum heat release rate becomes low if the number of the lateral faces affected by the instillation is smaller. 
$H-W / 2$ is the main variable for the duration of the maximum burning if no instillation occurs. The duration increases proportionally to $H-D / 2$ in case instillation occurs.

\section{REFERENCES}

1. The SFPE Handbook of Fire Protection Engineering Third Edition, pp.3-1-3-37, NFPA, 2002

2. Kakae N., Tuchihashi T. Tanaka Y. Ohmiya Y., and Harada K., "Influence of Combustible Dimension and Density on Heat Release Rate", Proceeding of the 76th Architectural Research Meetings, Kanto Chapter, pp.261-264, 2006 (in Japanese)

3. MIZUNO T., Sasagawa F., Horiuchi S., and Kawagoe K., "Burning Behaviour of Urethane Foam Mattresses ", Fire Science and Technology, Vol.1, pp.33-44, 1981

4. Nam D., Hasemi Y., Kagiya K. and Yoshida M., "Investigation of a Recent Large Wooden Gymnasium Fire -Part2 Experiment for the Estimation of the Mechanism to Flashover", Proceedings of JAFSE Annual Symposium 2002, pp.144-147, 2002 (in Japanese) 